\title{
Factores de riesgo relacionados con lupus eritematoso sistémico en población mexicana
}

A braham Zonana-N acach, MC, ${ }^{(1)}$ Leoncio Miguel Rodríguez-G uzmán, MC, ${ }^{(2)}$ Francisco Javier Jiménez-Balderas, MC, ${ }^{(1)}$ A dolfo Camargo-Coronel, MC, ${ }^{(1)}$ Jorge Escobedo-de la Peña, MC, ${ }^{(1)}$ Antonio Fraga, MC .1) $^{(1)}$

\section{Zonana-Nacach A, Rodríguez-Guzmán LM,} Jiménez-Balderas FJ, Camargo-Coronel A, Escobedo-de la Peña J, Fraga A.

Factores de riesgo relacionados con lupus eritematoso sistémico en población mexicana. Salud Publica Mex 2002;44:213-218.

El texto completo en inglés de este artículo está disponible en: http://www.insp.mx/salud/index.html

\section{Resumen}

Objetivo. Evaluar los factores de riesgo asociados con el desarrollo de lupus eritematoso sistémico en población mexicana. Material y métodos Estudio de casos y controles, efectuado en junio de 1996 en el Departamento de Reumatología del Hospital de Especialidades del Centro Médico N acional Siglo XXI (HE C MN ), del Instituto Mexicano del Seguro Social, de la Ciudad de México. Se estudiaron 130 pacientes (casos) que presentaban cuatro o más criterios de clasificación de lupus eritematoso sistémico (LES) y con una evolución de la enfermedad menor de cinco años. Los controles fueron pacientes hospitalizados por enfermedades agudas no auto inmunes. Fueron pareados 1:1 por edad y sexo; ambos grupos se evaluaron a través de una entrevista directa y la aplicación de un cuestionario estructurado. Se estudiaron los siguientes factores de riesgo: genéticos (historia familiar de LES o enfermedad de tejido conjuntivo), socio demográficos (raza, lugar de residencia, educación, ingreso mensual); hormonales (anticonceptivos orales, terapia hormonal de remplazo y ginecobstétricos); ambientales (productos para el cabello, cohabitación con perros, infecciones, alergias). El análisis estadístico fue hecho con razón de momios (RM) IC 95\% y regresión logística. Resultados El análisis estadístico multivariado mostró asociación con el antecedente familiar de LES (RM 4.2, IC 95\% 1.17-15.2) u otra ETC (RM 2.6, IC 95\% 1.15-4.5), uso de anticonceptivos orales por más de un año (RM 2.1, IC 95\% 1.13-4.3), faringoamigdalitis de repetición (RM 2.1, IC 95\%

\author{
Zonana-Nacach A, Rodríguez-Guzmán LM, \\ Jiménez-Balderas FJ, Camargo-Coronel A, \\ Escobedo-de la Peña J, Fraga A. \\ Risk factors associated with systemic lupus \\ erythematosus in Mexican population. \\ Salud Publica Mex 2002;44:213-218. \\ The English version of this paper \\ is available at: http://www.insp. $\mathrm{mx} / \mathrm{salud} / \mathrm{index} . \mathrm{html}$
}

\begin{abstract}
A bstract
Objective To assess risk factors associated with systemic lupus erythematosus (SLE) in the Mexican population. Material and Methods. A case-control study was conducted on June 1996, at the Reumathology C linic of Hospital de Especialidades del Centro Médico N acional Siglo XXI (HE CMN ), Instituto Mexicano del Seguro Social, in Mexico City. Cases were one hundred thirty subjects with four or more SLE criteria and disease evolution of \pm 5 years. Controls were hospitalized patients with acute diseases but without autoimmune diseases. Cases and controls were matched 1:1 by age and gender; both groups were evaluated by direct interview through a structured questionnaire. The following risk factors were assessed: genetic family history of SLE and connective tissue disease; so cioedemographic (ethnicity, geographic distribution, education, monthly income); hormonal (use of oral contraceptives, replacement therapy and gynecoobstetric background); environmental (use of hair products, living with dogs, bacterial/viral infections, and allergies). Statistical analysis consisted of odd ratios (OR) with 95\% confidence inter vals $\mathrm{Cl}$ ) and multivariate analysis using logistic regression. Results. The multivariate model showed association with family history of SLE (OR 4.2, CI 95\% 1.17-15.2), family history of connective tissue disorder (OR 2.6, CI 95\% 1.15-4.5), use of oral contraceptives for more than one year (OR 2.1, CI 95\% 1.13-4.3), repetitive pharyngitis (OR 2.1, Cl 95\% 1.18-3.6), and use of medications (OR 5.0 IC 95\% $1.62-21.6)$. N o association was found
\end{abstract}

(1) Departamento de Reumatología, Hospital de Especialidades Centro Médico N acional «Siglo XXI», Instituto Mexicano del Seguro Social (IMSS), México D.F., México.

(2) Coordinación de Salud Comunitaria, Instituto Mexicano del Seguro Social, México, D.F., México.

Fecha de recibido: 18 de mayo de 2001 - Fecha de aprobado: 5 de diciembre de 2001 Solicitud de sobretiros: D r. A braham Zonana-N acach. 143 1/2 D Avenue, Coronado CA, 92118, U.S.

Correo electrónico: zonanaa@ yahoo.com 
1.18 - 3.6) y fármacos (RM 5.0, IC 95\% 1.62 - 21.6). No hubo relación con factores socieconómicos, el uso de productos para el cabello, con asma o con antecedentes alérgicos. Conclusiones Factores genéticos como el antecedente heredo-familiar de LES o enfermedad de tejido conjuntivo en familiares de primer grado continúan siendo factores impo rtantes en el desarrollo de LES.O tros factores de riesgo como el uso de fármacos, uso de anticonceptivos orales, faringitis de repetición, posiblemente interactúan en un huésped genéticamente susceptible para el desarrollo de la enfermedad. El texto completo en inglés de este artículo está disponible en: http://www.insp.mx/salud/index.html

Palabras clave: lupus eritematoso sistémico; factores de riesgo; México with socieconomic status, hair dye products, asthma, or allergies. Conclusions. G enetic factors, such as family history of SLE and connective tissue disease in first-degree relatives, persist as important factors in the development of SLE. 0 ther factors, such as use of some drugs, oral contraceptives, and repetitive pharyngitis, may also favor the onset of disease in genetically susceptible hosts. The English version of this paper is available at:http://ww w.insp.mx/salud/ index.html

Key words: lupus erythematosus systemic; risk factors; Mexico
E 1 lupus eritematoso sistémico (LES), es una enfermedad autoinmune, inflamatoria, crónica y multisistémica de etiología desconocida, definida por sus características clínicas y por la presencia de autoanticuerpos dirigidos contra uno o más componentes del núcleo. La frecuencia de LES en gemelos monocigotos es de $25 \%$ comparada con $2 \%$ en gemelos dicigotos; ${ }^{1,2}$ aunque esto sugiere un papel importante de la herencia en el desarrollo de LES, ${ }^{3-7}$ el factor genético por sí solo no explica la etiología de la enfermedad. Otros factores de riesgo, incluyendo endócrinos, ${ }^{8-12}$ ambientales, ${ }^{13-17}$ infecciosos ${ }^{18-20}$ y fármacos ${ }^{21-24}$ pueden actuar sinérgicamente en el desarrollo de LES en un huésped genéticamente susceptible.

Existen pocos estudios con diseño epidemiológico que determinen la fuerza de asociación de algunos factores de riesgo y la presencia de LES. ${ }^{25-31}$ Tres de estos estudios ${ }^{26,28,30}$ tuvieron menos de 100 casos, lo cual limita el poder estadístico, y en otro ${ }^{26}$ los casos tenían más de 10 años de evolución, lo cual afecta la calidad de la información evaluada. El objetivo del presente trabajo fue determinar la asociación de factores de riesgo y el desarrollo de LES, con un estudio de casos y controles en población mexicana.

\section{Material y métodos}

Pacientes con LES, del Departamento de Reumatología del Hospital de Especialidades del Centro Médico Nacional (HE CMN) Siglo XXI, del Instituto Mexicano del Seguro Social (IMSS), han sido incluidos en una base de datos desde 1990. Estos pacientes por la severidad de su enfermedad ameritan atención en un hospital de tercer nivel. Generalmente, son referidos al HE CMN por las clínicas periféricas o los Hospitales Generales de Zona del Sur de la Ciudad de
México. Convencionalmente, dichos pacientes son evaluados cada tres meses en la consulta del Departamento de Reumatología.

Selección de casos. Pacientes con LES, con una duración de la enfermedad menor a cinco años y con cuatro o más criterios de clasificación de la Asociación Americana de Reumatología (ARA), actualmente el Colegio Americano de Reumatología (ACR), ${ }^{32}$ que acudieron a la consulta externa del Departamento de Reumatología de enero a junio de 1996.

Selección de controles. Los controles fueron seleccionados durante el verano de 1996. Fueron pacientes pertenecientes al HE CMN y que habían sido hospitalizados en los servicios de cirugía general o reconstructiva, otorrinolaringología u oftalmología, por enfermedades agudas no autoinmunes. Sólo fueron incluidos los pacientes sin antecedentes de enfermedades del tejido conjuntivo (ETC) u otra enfermedad crónica. Los criterios de elegibilidad para los controles fueron: estar dispuestos a participar en la entrevista y responder a un cuestionario. Los controles fueron pareados por edad ( \pm 5 años) y sexo con relación 1:1.

Medición de factores de riesgo. A través de una entrevista directa hecha por un epidemiólogo (LMRG), se aplicó un cuestionario estructurado el cual fue revisado y aprobado por los Comités de Investigación del Departamento de Coordinación de Salud Comunitaria y del HE CMN "Siglo XII". Fueron evaluadas las siguientes variables: antecedentes sociodemográficos, escolaridad, ingreso familiar mensual, tipo de ocupación (laborar dentro o fuera del hogar) y lugar de residencia durante los últimos diez años (zona urbana o rural); antecedentes en familiares de primer grado de LES u otras ETC como artritis reumatoide, esclerodermia, miopatías inflamatorias, síndrome de Sjö- 
gren primario, púrpura trombocitopénica idiopática y enfermedades autoinmunes del tiroides; antecedentes ginecobstétricos: tiempo de uso de anticonceptivos orales o terapia hormonal de remplazo, abortos espontáneos, desarrollo de LES durante el embarazo o posparto; antecedentes ambientales: uso de productos para el cabello (tintes, aerosol, gel o productos para el rizado del cabello), cohabitación con perros por un periodo mayor a $50 \%$ de la vida del sujeto. Se investigó la dosis y el consumo por un año o más de los siguientes fármacos antihipertensivos (procainamida, hidralazina alfametildopa, timolol, captopril, atenolol), anticonvulsivantes (carbamacepina, difenilhidantoína), antibióticos (ciprofloxacina, isoniazida, minociclina), inmunomodulares (interleucina-2, interferón alfa y Dpenicilamina), antinflamatorios no esteroideos (sulfazalazina, fenilbutazona); infecciones bacterianas como faringo amigdalitis de repetición (cuatro o más al año, acompañadas de fiebre, ataque al estado general y mejoría con el uso de antibióticos) y tuberculosis; infecciones virales (sarampión, rubéola, parotiditis, hepatitis, herpes simple, herpes zoster y varicela); cuadros alérgicos (asma, rinitis alérgica, conjuntivitis crónica, urticaria, alergia secundaria a drogas o alimentos), evaluados previamente por un especialista y ocurridos al menos un año antes del diagnóstico del lupus.

Se construyeron tablas de contingencia para evaluar asociaciones entre los factores de riesgo de los casos y controles. Para las variables categóricas se utilizó $\chi^{2}$ y se obtuvieron las razones de momios (RM) con sus intervalos de confianza de 95\% (IC 95\%). Asimismo, se aplicó análisis de regresión logística para obtener la razón de momios con IC 95\% y estimar la asociación de factores de riesgo para el desarrollo de LES. Los factores de riesgo fueron ajustados por sexo.

\section{Resultados}

Fueron incluidos en el estudio 130 casos e igual número de controles. Veintiuno (16\%) tuvieron una duración de LES de un año o menos; 82 (63\%) entre 2-3 años y $27(20 \%)$ entre $4-5$ años. Como era de esperarse, la distribución por sexo, edad, educación e ingreso mensual familiar de los casos y controles fue similar, sin encontrarse significancia estadística. El $94 \%$ fueron mujeres, con una edad promedio para los casos de $27 \pm 8$ y de $30 \pm 6$ años para los controles; $52 \%$ de los casos y $49 \%$ de los controles tuvieron una educación $\geq 12$ años; el ingreso mensual familiar fue similar para los casos (5 700 pesos) y los controles (5 430 pesos ).

La única variable sociodemográfica asociada con LES fue educación. Pacientes con una escolaridad ma- yor de nueve años tuvieron una RM de 2.18 (IC 95\% 1.2-3.9). No hubo asociación con el antecedente de laborar fuera del hogar (RM 1.89, IC 95\% 0.92-3.41), el ingreso mensual familiar (RM 1.3, IC 95\% 0.5-3.3), pertenecer a población urbana ( $R M 0.6$, IC $95 \% 0.2-1.7)$ o rural ( $R M$ 0.8, IC 95\% 0.5-1.4). Asimismo, no se encontró asociación con el uso de tintes (nunca haberse teñido el cabello, o con tinción mensual o anual), con la frecuencia de uso de fijadores o con el número de veces del rizado del cabello o con el antecedente de alergias (cuadro I).

Hubo asociación entre LES y el antecedente de lupus en familiares de primer grado, RM 5.0 (IC 95\% 1.67-21.6), y el antecedente familiar de ETC RM 2.0 (IC 95\% 1.23-3.5). El uso de anticonceptivos orales durante un año o más tuvo un riesgo dos veces mayor de desarrollar LES, RM 2.0 (IC 95\% 1.81-5.3) y de cinco veces mayor con el antecedente de la ingesta de isoniacida, alfametildopa y carbamazepina, RM 5.0 (IC 95\% 1.67-21.6). En el análisis bivariado otras variables asociadas con el desarrollo de LES fueron amigdalitis de repetición, RM 2.1 (IC 95\% 1.21-3.6) y cohabitación con perros, RM 1.91 (IC 95\% 1.16-3.2).

Las variables retenidas en el análisis multivariado fueron antecedente familiar de LES o ETC, amigdalitis de repetición, utilización de fármacos y de anticonceptivos orales por un año o más (cuadro II).

\section{Discusión}

Nuestra investigación tiene varias cualidades: es el primer estudio realizado sobre factores de riesgo en el desarrollo de LES en población mexicana; a diferencia de otros estudios epidemiológicos, en el nuestro los casos tuvieron un diagnóstico temprano de LES, promedio tres años, $y$, finalmente, se estudiaron variables no evaluadas previamente como las socioeconómicas y el uso de fármacos.

El LES es un modelo para el estudio de otras enfermedades autoinmunes; su etiología es multifactorial e involucra uno o más factores ambientales, los cuales actúan sobre un huésped genéticamente susceptible. Hochberg y colaboradores, ${ }^{4}$ en un estudio de casos y controles, determinaron la presencia de LES en hijos con padres con o sin lupus. En los casos se presentó LES en $10.4 \%$ de los hijos, en tanto en los controles, sólo $1.3 \%$ tuvo lupus, con un riesgo relativo de 8. Para observar una relación genética más estrecha, se han realizado estudios en gemelos monocigotos y dicigotos, concordando la presencia de la enfermedad en 24 y $2 \%$, respectivamente. ${ }^{3}$ Asimismo, el antecedente en familiares de primer grado de otras ETC como artritis reumatoide, esclerodermia y polimiosi- 


\section{Cuadro I \\ ANÁLISIS BIVARIADO DE FACTORES DE RIESGO relacionados con Lupus Eritematoso Sistémico. Departamento de Reumatología, Centro Médico Nacional Siglo XXI, México, 1996}

Casos Controles RM (IC 95\%) $n=130 \quad n=130$

\begin{tabular}{llrrr}
$\begin{array}{l}\text { Antecedentes } \\
\text { heredo-familiares } \\
\text { Lupus eritematoso sistémico }\end{array}$ & Sí & 15 & 3 & 5.0 (1.67-21.6) \\
\hline & No & 115 & 127 & \\
\hline Enfermedad del tejido conjuntivo & Sí & 53 & 34 & 2.0 (1.23-3.5) \\
\hline & No & 77 & 96 &
\end{tabular}

Uso de anticonceptivos

\begin{tabular}{lrrrr} 
orales (AO) & Sí & 38 & 14 & $1.96(1.45-3.9)$ \\
\hline Tiempo uso AO & No & 92 & 116 & \\
N unca & & 92 & 116 & $0.28(0.14-0.55)$ \\
\hline Menos de un año & 14 & 9 & $1.86(0.75-4.9)$ \\
\hline 1 o más años & 24 & 5 & $2.0(1.81-5.3)$ \\
& & & \\
Uso de tintes & & & \\
$\quad$ unca & 89 & 81 & $1.3(0.78-2.1)$ \\
\hline Anual & 26 & 34 & $0.67(0.37-1.2)$ \\
\hline Mensual & 15 & 15 & $1.0(0.46-2.1)$
\end{tabular}

Uso de fijadores

N unca

\begin{tabular}{llll} 
Semanal o mensual & 67 & 66 & $1.0(0.63-1.6)$ \\
\hline Diario & 21 & 34 & $0.54(0.29-1.0)$ \\
\hline
\end{tabular}

Uso de permanentes

\begin{tabular}{lcrc}
$N$ unca & 46 & 54 & $0.72(0.43-1.1)$ \\
\hline 1 a 5 veces & 51 & 47 & $1.1(0.79-1.4)$ \\
\hline 6 o más veces & 33 & 29 & $1.1(0.66-2.0)$
\end{tabular}

Cohabitación con perros

\begin{tabular}{llll} 
Sí & 72 & 51 & $1.91(1.16-3.2)$ \\
\hline No & 58 & 79 &
\end{tabular}

Amigdalitis de repetición

\begin{tabular}{llll} 
Sí & 66 & 44 & $2.1(1.21-3.6)$ \\
\hline No & 64 & 86 &
\end{tabular}

Uso de fármacos

\begin{tabular}{lrrr} 
Sí & 16 & 4 & $5.0(1.67-21.6)$ \\
\hline No & 114 & 126 &
\end{tabular}

tis ha sido asociado con un mayor riesgo para el desarrollo de LES, que varía de 2.3 a 20.25,26,29

Está bien reconocido el efecto del sexo sobre la susceptibilidad para ciertas enfermedades autoinmunes. En el LES existe un claro efecto de las hormonas

\section{Cuadro II \\ Análisis MUltivariado de factores de RIESGO asociados con Lupus Eritematoso Sistémico.* Departamento de Reumatología, Centro Médico Nacional Siglo XXI, México, 1996}

\begin{tabular}{lccrcc} 
& \multicolumn{2}{c}{ Población total } & & \multicolumn{2}{c}{ M ujeres } \\
\cline { 2 - 3 } & RM & IC $95 \%$ & & RM & IC 95\% \\
AHF de LES & & & & & \\
\hline AHF de ETC & 4.2 & $1.17-15.2$ & & 3.8 & $1.02-14.4$ \\
\hline Faringo amigdalitis & 2.6 & $1.15-4.5$ & & 2.6 & $1.15-4.6$ \\
\hline Fármacos & 2.1 & $1.18-3.6$ & 2.8 & $1.13-3.6$ \\
\hline Anticonceptivos orales & 5.0 & $1.62-21.6$ & 4.1 & $1.14-15.0$ \\
\hline & & & 2.1 & $1.13-4.3$
\end{tabular}

* Los factores de riesgo se ajustaron por sexo

sexuales femeninas en el desarrollo y evolución del LES. Durante la edad reproductiva el lupus afecta a nueve mujeres por cada hombre, en tanto que antes de la pubertad esta proporción es tan sólo de tres a uno, y después de la menopausia la frecuencia de lupus es similar en mujeres y hombres. El uso de anticonceptivos orales en pacientes con LES puede contribuir al desarrollo de la enfermedad u ocasionar exacerbación de la misma. Nuestro estudio demostró un riesgo dos veces mayor con el uso de anticonceptivos orales solamente cuando éstos se usaron por un año o más. Sánchez-Guerrero y colaboradores ${ }^{8}$ encontraron un ligero incremento de riesgo ( $R R 2.1$, IC 95\% 1.4-1.4) para presentar LES en mujeres con uso previo o corriente de anticonceptivos orales, el riesgo fue directamente proporcional al tiempo de uso de anticonceptivos. Sin embargo, esto no ha sido corroborado por otros. ${ }^{25,31}$ Actualmente las mujeres con LES tienen una supervivencia similar a las mujeres sanas, por lo que hay un número mayor de mujeres posmenopáusicas y LES en riesgo de osteoporosis. Los pocos estudios realizados para evaluar reactivación de LES por uso de terapia hormonal de remplazo no han mostrado un riesgo mayor de exacerbación. ${ }^{33}$

Se ha descrito la asociación de LES principalmente con el uso de fármacos antihipertensivos, anticonvulsivantes, antifímicos y antibióticos..$^{21-24}$ Sin embargo, en estudios epidemiológicos previos ${ }^{25-31}$ no se había evaluado esta variable. En nuestro estudio hubo un riesgo cinco veces mayor ( $R M$ 5.0, IC 95\%1.6-21.6) al evaluar en conjunto el antecedente de uso a dosis convencionales de isoniazida, alfametildopa o carbamazepina y no hubo asociación con el uso de otros medicamentos. Los productos para el cabello como aerosoles, tintes y permanentes han sido estudiados 
como posibles factores de riesgo relacionados con el desarrollo de LES, ya que contienen aminas aromáticas similares a algunas drogas como hidralazina o procainamida, que pueden inducir lupus. En 1986, Freni-Titulaer y colaboradores ${ }^{26}$ encontraron asociación entre el antecedente de uso de productos para el cabello y LES. Sin embargo, ni nuestro estudio ni otros ${ }^{13,34}$ han confirmado dicha asociación.

Se ha demostrado que productos bacterianos pueden jugar un papel en la patogénesis del lupus. Caballo y colaboradores ${ }^{35}$ encontraron un aumento en la activación de células $B$, niveles elevados de anticuerpos anti-DNA y el desarrollo de glomerulonefritis proliferativa difusa, posterior a la aplicación de productos de lipopolisacáridos bacterianos a ratones NZB/W. Sin embargo, el significado de esto y su relación con el lupus en humanos no ha sido determinado. La prevalencia de faringitis aguda en la población general varía entre 15 y 30\% ${ }^{36}$ dependiendo de la población estudiada, la cual es similar a la observada en nuestro trabajo. Aunque encontramos un aumento de riesgo de dos veces más para desarrollar LES en sujetos con el antecedente de cuadros faringoamigdalinos de repetición, esto debe tomarse con cautela ya que puede haber sesgo de memoria en su medición, debido a su temporalidad. No encontramos asociación entre infecciones exantemáticas como sarampión, rubéola, parotiditis, varicela u otras infecciones virales (hepatitis, herpes simple o herpes zoster) y LES como ha sido reportado por Strom y colaboradores, ${ }^{25}$ quienes encontraron que la presencia de herpes zoster previo al diagnóstico de LES fue asociada con el desarrollo del mismo (RM 4.7, IC 95\% 1.1-21.7) .

Aunque no hubo asociación con indicadores de nivel socioeconómico como educación, empleo e ingreso familiar, estas variables no habían sido evaluadas. ${ }^{25-31}$ Nosotros no evaluamos otros factores que se han asociado con el desarrollo de lupus como el antecedente de trabajar en un medio ambiente "polvoriento" $^{\prime 37}$ (RM 3.9, IC 95\% 1.6-9.7), consumo frecuente de carnes rojas ${ }^{30}$ (RM 3.3, IC 95\%1.10-10.2), irregularidad en el periodo menstrual ${ }^{30}$ (RM 3.7, IC 95\% 1.4-10.0), exposición prolongada a emisiones industriales, ${ }^{17}$ contaminación del agua con tricloroetileno ${ }^{38}$ y exposición a sílice. ${ }^{39}$

En conclusión, nuestro estudio mostró que el antecedente heredo-familiar de lupus y de ETC en familiares de primer grado fue el factor más asociado con el desarrollo de LES. Asimismo, otros factores de riesgo como el uso de ciertos fármacos, faringoamigdalitis de repetición y el uso de anticonceptivos orales por más de un año, probablemente interactúan sinérgicamente en un huésped genéticamente susceptible al desarrollo de la enfermedad. Se desconoce cómo los factores geneticos interactúan con otras variables en la presencia de la enfermedad. Sin embargo, actualmente existe un mejor conocimiento y entendimiento de los factores que tienen influencia en la inmunorregulación y el desarrollo de autoinmunidad en el lupus. Son necesarios más estudios para que se demuestre un reconocimiento de los factores que interactúan en los estadios iniciales de la patogénesis del LES.

\section{Referencias}

1. Fessel W F. Epidemiology of systemic lupus erythematosus. Rheum D is Clin N orth Am 1988;14:15-23.

2. D eapen D, Escalante A, W einrib L, H orwitz D, Bachman B, Roy-Burman $P$ et al. A revised estimate of twin concordance in systemic lupus erythematosus. Arthritis Rheum 1992;35:311-318.

3. Lawrence JS, Martins CL, D rake GL.A family survey of lupus erythematosus heritability.J Rheumatol 1987;14:913-921.

4. Hochberg MC, Flosheim F, Scott J, Arnett J. Familial aggregation of systemic lupus erythematosus. Am J Epidemiol 1985;122:959-966.

5.Alvaro-G arcía JM, H umbríaA, García-Vicuña R,ArizaA , G arcía-Vadillo A, Laffón $A$. Systemic lupus erythematosus and tetrasomy-X. J Rheumatol 1989;16:1486-1488.

6. Seuchter SA, Knapp M, Hartung K, Coldewey R, Kalden JR, Lakmek HJ et al. Testing the association in SLE families. Genet Epidemiol 1991;8: 409-416.

7. W akeland EK,W andstrat AE, Liu K, Morel L. Genetic dissection of systemic lupus erythematosus. Curr 0 pin Immunol 1999;11:701-707.

8. Sánchez-Guerrero J, Karlson EW, Liang MH, Hunter DJ, Speicer FE, Golditz GA. Past use of oral contraceptives and the risk of developing systemic lupus erythematosus. Arthritis Rheum 1997;40:804-808.

9. Rood MJ, Van der Velde EA, Ten Cate R, Breedveld FC, Huizinga TW. Female sex hormones at the onset of systemic lupus erythematosus affect survival. Br J Rheumatol 1998:37:1008-1010.

10. Liang MH , Karlson EW. Female hormone therapy and the risk factor of developing or exacerbating systemic lupus erythematosus or rheumatoid arthritis. Proc Assoc Am Physicians 1996;108:25-28.

11. Mier CR, Sturkkenboom MC, C ohen AS, Jick H. Postmenopausal estrogen replacement therapy and the risk of developing systemic lupus erythematosus or discoid lupus. J Rheumatol 1998;25:1515-1519.

12. Lahita RG.The role of sex hormones in systemic lupus erythematosus. Curr O pin Rheumatol 1999;11:352-356.

13. Petri M, Allbritton J. Hair product use in systemic lupus erythematosus. A case-control study. Arthritis Rheum 1992;35:625-629.

14. Love LA. N ew environmental agents associated with lupus-like disorders. Lupus 1994;3:467-471.

15. Mayes MD. Epidemiologic studies of environmental agents and systemic autoimmune diseases. Environ Health Perspect 1999;107(Suppl 5):743-748.

16. Cooper GS, D o oley MA, Treadwell EL, St. Clair EW, Parks CG, G ilkeson GS. Hormonal, environmental, and infections risk factors for developing systemic lupus erythematosus. Arthritis Rheum 1998;41:1714-1724. 17. Kardestuncer T, Frumkin H. Systemic lupus erythematous in relation to environmental pollution in an African-American community in $\mathrm{N}$ orth Georgia. Arch Environ Health1997;52:85-90.

18. Granholm N A, Cavallo T. Autoimmunity, polyclonal B-cell activation and infection. Lupus 1992;2:163-174. 
19. G ranholmy N A, C avallo T. Enhancement of renal disease in BX SB lipus prone mice after prior exposure to bacterial lipopolysaccharide. Lupus 1995; 4:339-347.

20. A cha-O rbea H. Bacterial and viral superantigens: Role in autoimmunity? Ann Rheum D is 1993; 52(Suppl 1):S6-S16.

21. Hess EV. Role of drugs and environmental agents in lupus syndromes. Curr 0 pin Rheumatol 1992;4:688-692.

22. Salazar-Páramo M, Rubín RL, G arcía de la Torre J. Systemic lupus erythematosus induced by isoniazid. Ann Rheum D is 1992;51:1085-1087.

23. Fritzler MJ. Drug recently associated with lupus syndromes. Lupus 1994;3:455-459.

24. Montoya-C abrera MA, Sauceda-G arcía JM, Escalante-G alindo P, LópezMorales E.Thallium poisoning wich stimulated systemic lupus erythematosus in a child. Gac Med Mex 1991;127:333-336.

25. Strom BL, Reidenberg MM, W est S, Sim ES, Freundlich B, Stolley PD. Shingles, allergies, family history, oral contraceptives, and other potential risk factors for systemic lupus erythematosus. Am J Epidemiol 1994;140: 632-642.

26. Freni-Titulaer LW, Kelley D B, G row AG , M CKinleyTW, A rnett FC , Hochberg MC. Connective tissue disease in Southeastern G eorgia:A case-control study of etiologic factors. Am J Epidemiol 1989;130:404-409.

27. Hardy CJ, Plamer BP, Muir KR, Sutton AJ, Powell RJ. Smoking history, alcohol consumption, and systemic lupus erythematosus: A case-control study.Ann Rheum D is 1998:57:451-455.

28. Benoni $C, N$ ilson $\AA$, N ived 0 . Smoking and inflammatory bowel disease: Comparison with systemic lupus erythematosus:A case-control study. Scand J Gastroenterol 1990;25:751-755.

29. N agata C, Fujita S, Iwata H, KurosowaY, Ko bayashi K, Ko bayashi M et al. Systemic lupus erythematous: A case-control epidemiologic study in Japan. Int J Dermatol 1995;34:333-337.

30. Minami Y, Sasaki T, Komatsu S, N ishikori M, Fukao A, Yoshinaga K et al. Female systemic lupus erythematosus in Miyagi Prefecture, Japan:A case- control study of dietary and reproductive factors. Tohoku J Exp Med 1993;169:245-252.

31. Grimes $D A$, LeBolt $S A, G$ rimes $K R, W$ ingo PA. Systemic lupus erythematosus and reproductive function:A case control study. Am J 0 bstet Gynecol 1985;153:179-186.

32.Tan EM, C ohen AS, Fries JF, Masi AT, M CShane DJ, Rothfield N F et al.The 1982 revised criteria for the classification of systemic lupus erythematosus (SLE). Arthritis Rheum 1982;25:1271-1277.

33. Arden N k, Lloyd M, Spector TD, Hughes GRV. Safety of hormone replacement therapy (ERT) in systemic lupus erythematosus. Lupus 1994;3: 11-13.

34. Sánchez-Guerrero J, Karlson EW, Colditz GA, Hunter DJ, Speizer FE, Liang $\mathrm{MH}$. Hair due products and the risk of developing systemis lupus erythematosus. Arthritis Rheum 1996;39:657-662.

35. Cavallo T, Granholm N A. Bacterial lipopolysaccharide transform mesangial into prolifer ative lupus nephritis without interfering with processing of pathogenic immune complexes in NZB/W mice. Am J Pathol 1990; 971-978.

36. Bisno AL.A cute pharingitis. N Engl J Med 2001;344:205-211.

37. N agata C, Yoshida H, Fujita S, KomuraY, Inaba R, Iwata H et al. A casecontro study of SLE based on patients receiving finnacial aid for tretament. Recent progress of epidemiologic study of intractable diseases in Japan. Tokio: Ministery of Health and W elfare of Japan, 1992:113-116.

38. Kilburn KH,W arshaw RH. Prevalence of symptoms of systemic lupus erythematosus (SLE) and of fluorescent antinuclear antibodies associated with chronic exposure to trichloroethylene and other chemicals in well water. Environ Res 1992;57:1-9.

39. Lüthke K, Conrad K, Mehlhorn J, D örmer T, Frank KH. Systemic lupus erythematosus after heavy exposure to quartz dust in uranium mines: Clinical and serological characteristics. Lupus 1996;6:62-69. 